\title{
Influence of Spin Projection Noise on the Sensitivity of Cs Atom Magne- tometer
}

\author{
Wang Feng", Li Hongmei, Liu Bingsheng, Ren changyu, and Shi Hongxin
}

College of Science, Heilongjiang University of Science and Technology, Harbin 150022, China

\begin{abstract}
This article introduces the influence of laser's spin projection noise on the sensitivity of Cs atom magnetometer and concludes that the theoretical value of the spin projection noise is about $1.23 \mathrm{fT} \cdot \mathrm{Hz}^{-1 / 2}$ under the optimization conditions of the atom steam chest, namely temperature as $105^{\circ} \mathrm{C}$, volume as $0.0785 \mathrm{~cm}^{3}$ and molecular density as $1.7 \times 10^{13}$ $\mathrm{cm}^{-3}$. Such research has provided us with the necessary basis for the experimental study on Cs atom magnetometer.
\end{abstract}

Keywords: Cs vapor magnetometer, Atom Magnetometer Sensitivity, Spin projection noise, Optical Pumping.

\section{INTRODUCTION}

The accurate weak magnetic field detection technology plays an irreplaceable important role in various fields such as medical science [1-5], military [1-5], physical geography and industry. Therefore, improving magnetometer sensitivity has become the research direction of the science researchers since 1950s. In 1960s, the superconducting quantum magnetometer was made from superconducting materials on the basis of Josephson Effect, with extremely high measuring sensitivity able to reach $10^{-15} \mathrm{~T} \cdot \mathrm{Hz}^{-1 / 2}$, but it also had obvious limitation that it could only work under liquid nitrogen environment at low temperature. Cs atoms are used as the working medium in order to achieve the self-exchange relaxationfree (SERF) atom magnetometer at about $100^{\circ} \mathrm{C}$. Under the working mechanism of this kind of magnetometer, the spin interaction rate is greatly more than Larmor precession rate $[6,7]$. Therefore, the relaxation depending on the spin interaction crash is eliminated. At present, the scientists in such countries as U.S., Russia are positively researching and developing the self-exchange relaxation-free atom magnetometer. In 2004, NIST team developed the atom magnetometer device with SERF-k sensitivity able to reach $50 \mathrm{pT} \cdot \mathrm{Hz}^{-1 / 2}[8]$, and such device has been improved for several times, with the sensitivity able to approach to $10 \mathrm{fT} \cdot \mathrm{Hz}^{-1 / 2}$ [9] in 2009 . Afterwards, Professor M.V. Romalis and his student Doctor S.J. Seltzer developed SERF-K atom magnetometer with the sensitivity of $1 \mathrm{pT} \cdot \mathrm{Hz}^{-1 / 2}[10]$ and able to work under the unshielded condition. Additionally, the factors influencing the sensitivity of SERF-Cs include incident intensity $[8,9]$, temperature $[8,9]$ of Cs atom steam chest, pressure [11] of Cs atom steam chest, spin projection noise [12], etc. Therein, the restraining influence of spin projection noise on bandwidth is put forward in document [12], but the influence of temperature, pressure, volume and molecular density of the

\footnotetext{
*Address correspondence to this author at the College of Science, Heilongjiang University of Science and Technology, Harbin, Heilongjiang, 150027, P.R. China; Tel: +86 0451 88036306; Fax: +86 045188036306 ; E-mail: wangfeng5678@163.com
}

atom steam chest, spin projection, etc. on Cs atom magnetometer sensitivity is not mentioned. In this article, the numerical calculation is adopted to analyze the factors influencing SERF-Cs atom magnetometer sensitivity, and meanwhile, the relation between the spin projection noise and the laser frequency detuning is numerically calculated.

\section{WORKING PRINCIPLE OF CS ATOM MAGNE- TOMETER}

SERF-Cs atom magnetometer is composed of pumping laser, probe laser, Cs atom steam chest and signal detection system. The schematic diagram is as shown In Fig. (1).

The pumping light is the right-handed circularly polarized light working at $894 \mathrm{~nm}$ (corresponding to $\mathrm{D}_{1}$ transition of Cs atoms). The light spots with the diameter of $4 \mathrm{~mm}$ spread along the positive direction of $\mathrm{Z}$-axis and enable the atoms to generate the transition of $\left|6^{2} s_{1 / 2}, m_{s}=-1 / 2\right\rangle \rightarrow\left|6^{2} p_{1 / 2}, m_{s}=1 / 2\right\rangle$. Due to the unstable excited state, electrons spontaneously radiate back to the stationary state. However, under the continuous action of the pumping light, all atoms are under the state of $\left|6^{2} s_{1 / 2}, m_{s}=1 / 2\right\rangle$ (without considering the transverse relaxation).

Macroscopically, all Cs atoms are directionally aligned along $\mathrm{Z}$-axis to generate magnetic moment, in other words, atoms are magnetized along $Z$-axis. In case of applying a weak magnetic field $\mathrm{B}$ along $\mathrm{Y}$-axis, the magnetic moment will generate Larmor procession in XOZ plane around $\mathrm{Y}$ axis. Due to the existence of transverse relaxation, the procession and the relaxation of the atoms can reach balance and generate an equivalent magnetic moment along $\mathrm{X}$-axis. The change cycle of this magnetic moment is proportional to $\mathrm{B}$, and then the magnetic moment along $\mathrm{X}$-axis is equivalent to the population difference of the spin quantum number on the two energy levels projected along $\mathrm{X}$-axis. When the probe laser (linearly polarized light spreading along $\mathrm{X}$-axis 


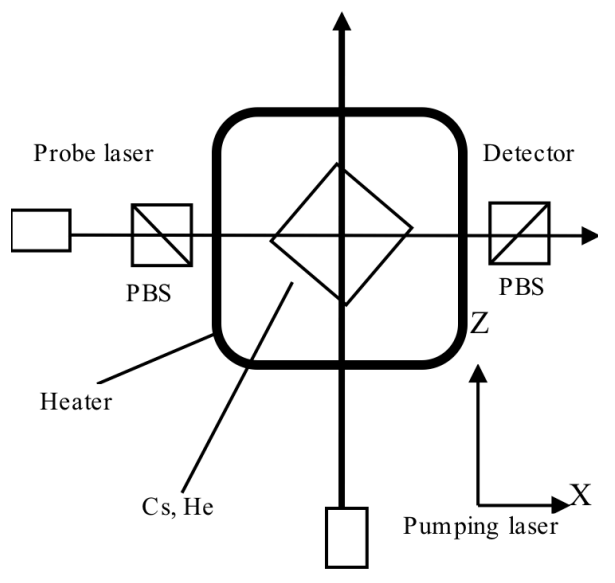

Fig. (1). Schematic diagram of SERF-Cs atom magnetometer.

and polarizing along $Z$-axis) with the wavelength detunes to $\mathrm{D}_{2}$ resonant frequency by $0.2 \mathrm{~nm}$ interacts with Cs atoms, the energy level population number will cause the left-handed and right-handed polarized light to sense different atomic dispersion parameters. In this way, after the probe laser passes through Cs bubble, the polarization plane thereof will have deflection. Therefore, the strength of the magnetic field $\mathrm{B}$ applied to $\mathrm{Y}$-axis can be calculated through measuring the rotation angle of the probe laser at the polarization direction, to achieve the purpose of weak magnetic field detection. In this experiment, the polarization elements along $\mathrm{Z}$-axis and $\mathrm{Y}$-axis are separated by the polarization beam splitter (PBS), and then the rotation angle of the polarization plane can be obtained through the calculation on the light intensity of the two arms of PBS.

\section{THEORETICAL ANALYSIS OF SPIN PROJEC- TION NOISE}

According to the interaction of light and atoms, we can know that the magnetometer sensitivity is influenced by the basic process of the spin projection noise which restrains the further improvement of SERF-Cs atom magnetometer sensitivity. Now, we theoretically analyze the source and the production mechanism thereof and meanwhile calculate the influence degree of the noise.

The spin projection noise is the performance of the corpuscular property of the quantized laser field, and such noise is different from the noise of the classic theoretical light field due to the limitation of Heisenberg uncertainty relation. In the classic theoretical light field, all noises can be regarded as the physical noise source to be treated and can be eliminated in principle. However, the spin projection noise is the physical quantity for quantum optics description and cannot be eliminated.

In the atom magnetometer, the photons of the probe laser beam continuously measure Fx. If the repeated measuring speed of a specific atom is faster than the transverse relaxation time $T_{2}$, then the measurement has correlation [13].
An expression is put forward in reference [13], and after the continuous measurement period, the uncertainty of Fx can be expressed as:

$$
\left(\frac{\left\langle\delta F_{x}\right\rangle}{\delta F_{x}}\right)^{2}=\frac{2}{t} \int_{0}^{t}(1-\tau / t) K(\tau) d \tau
$$

The correlation function of the spin time is defined according to the relaxation time $\mathrm{T}_{2}$, namely:

$$
K(\tau)=\exp \left(-\tau / T_{2}\right)
$$

Since the experiment is focused on the long-term measurement, $\mathrm{t}$ is assumed to be infinity when $\mathrm{t}$ is greatly more than $\mathrm{T}_{2}$ :

$$
\left\langle\delta F_{x}\right\rangle=\delta F_{x} \sqrt{\frac{2 T_{2} q(P)}{t}}
$$

The polarization root-mean-square uncertainty of the electron spin along $\mathrm{X}$-axis is as follows:

$$
\begin{aligned}
& \langle F\rangle=\frac{q(P)}{2} P \\
& \delta p_{x}^{r m s}=\frac{\delta\left\langle F_{x}\right\rangle_{t}}{F_{z}}=\frac{2}{q(p)} \delta\left\langle F_{x}\right\rangle_{t}=\sqrt{\frac{2 F_{z} T_{2}}{N}}
\end{aligned}
$$

In the formula, $\mathrm{N}=\mathrm{nV}$, wherein $\mathrm{n}$ is Cs atomicity density and $\mathrm{V}$ is the volume of Cs steam chest. In all electrons, the polarization can be defined as the overall average expected value of the electron spin orientation, namely $P_{z}^{e} \equiv 2\left\langle S_{z}\right\rangle$. Zero polarization means that the overall spin orientation is random. Additionally, the complete polarization along $\hat{z}$ direction is expressed by $P_{Z}^{e}=1$

$$
\left.\begin{array}{c}
\left\langle p_{x}\right\rangle=\frac{F_{x}}{F_{z}}=\frac{1}{2} \gamma B T_{2} \\
F_{z} \approx I+\frac{1}{2}=4
\end{array}\right\}
$$

The spin projection noise influences the sensitivity of the atom magnetometer due to the polarization uncertainty caused by the angular momentum uncertainty. The relation between the noise and the polarization is as follows:

$$
\frac{\delta B_{s p n}}{B}=\frac{\delta p_{x}}{p_{x}}
$$

According to formulae (5), (6) and (7), we can deduce the spin projection noise generated due to the influence of the uncertainty on the probe laser. The expression of the spin projection noise is as follows:

$$
\delta B_{s p n}=\frac{1}{\gamma} \sqrt{\frac{2}{n V T_{2}}}
$$




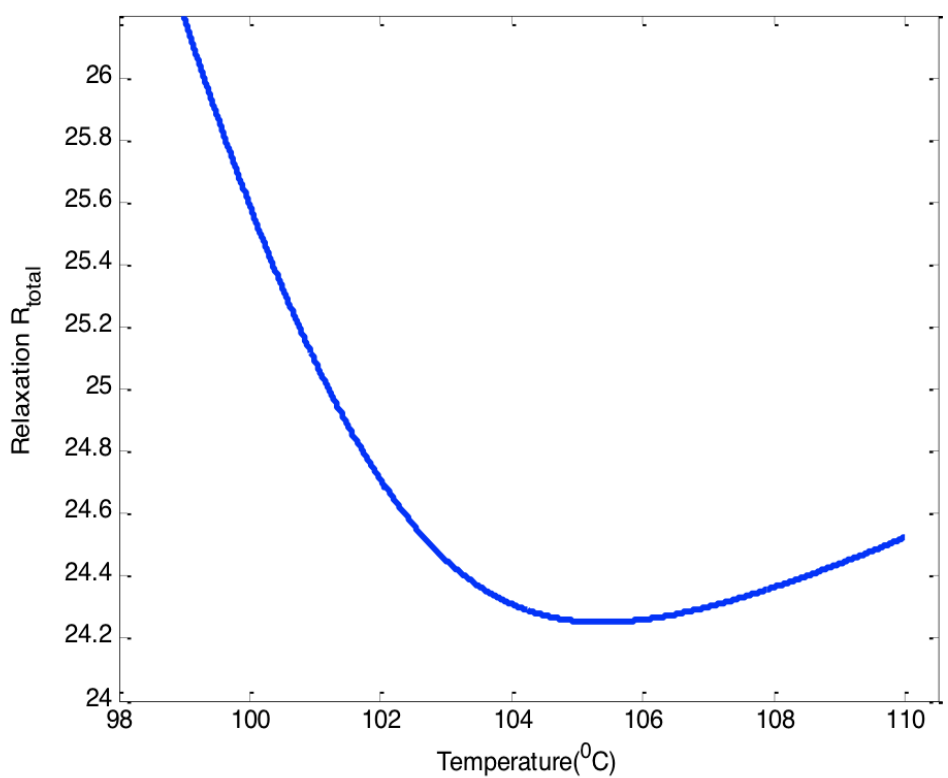

Fig. (2). The relation between the total transverse relaxation coefficient of Cs atom magnetometer and the temperature.

In the formula, $T_{2}=\frac{1}{R_{\text {total }}}$, wherein $R_{\text {total }}$ is the overall transverse relaxation coefficient of Cs atoms; under the selfexchange relaxation-free condition, the expression is as follows:

$$
R_{\text {total }} \approx R_{\text {sd }}^{\text {collisions }}+R_{p}(z)+R_{D}
$$

The physical meanings of these symbols are respectively shown as follows; $R_{s d}^{\text {collisions }}:$ Cs spin-destruction relaxation coefficient; $R_{D}$ : Cs scattering relaxation coefficient; $R_{p}(z)$ : Cs atom relaxation coefficient caused by pumping light (also called pumping coefficient). We consider the approximation of Cs spin-destruction relaxation coefficient $R_{s d}^{\text {collisions }} \approx 240 s^{-1}$, and then Cs atom relaxation coefficient caused by pumping light $R_{p}(z)$ is expressed as follows:

$$
R_{p}(z)=R_{s d} W\left[\frac{R_{p 0}}{R_{s d}} \cdot e^{R_{p 0} / R_{s d}} \cdot e^{-n \sigma\left(v_{0}\right) z}\right]
$$

In the formula, $R_{p 0}$ is the pumping coefficient of the pumping laser rate at the position $\mathrm{x}=0$ in the space, $\sigma\left(v_{0}\right)$ is the atomic absorption cross section of the pumping light at resonance, and $\mathrm{W}[\mathrm{x}]$ is the inverse function of Lambert $\mathrm{W}$ function [14].

Another factor is the scattering relaxation coefficient $R_{D}$ of $\mathrm{Cs}$ atom container wall and the expression of $R_{D}$ is as follows [15]:

$$
R_{D}=q(p) \sigma_{D} \sqrt{\frac{273+T}{273}} \frac{1}{p_{H e}}\left(\frac{\pi}{a}\right)^{2}
$$

In the formula, $\sigma_{D}=2.4 \times 10^{-23} \mathrm{~cm}^{2}$ is the scattering across section of Cs atoms in $\mathrm{He}$ buffer gas; $\mathrm{T}, \mathrm{P}_{\mathrm{He}}$ and $\mathrm{a}=1.25 \mathrm{~cm}$ are respectively the gas temperature, the pressure of He buffer gas and the radium of Cs bubble. The relation between the total transverse relaxation coefficient of Cs atom magnetometer and the temperature is as shown in Fig. (2).

The transverse relaxation time is related to the probe laser frequency, so we can obtain the relation between the spin projection noise and the probe laser frequency detuning, as shown in Fig. (3).

The spin projection noise expression (8) shows that the noise intensity is not depended on the moderation factor of the nucleus but is influenced by the molecular density, the volume and the total relaxation. According to Fig. (4), the larger the molecular density, the smaller the spin projection noise; according to Fig. (5), the larger the volume of Cs steam chest, the smaller the spin projection noise. In conclusion, the noise intensity is inversely proportional to the volume, the molecular density and the total relaxation coefficient.

\section{CONCLUSION}

We have analyzed Cs atom magnetometer and concluded that under the optimization condition of $\mathrm{Cs}$ atom steam chest, namely temperature as $105 \mathrm{C}$, volume as $0.0785 \mathrm{~cm}^{3}$ and molecular density as $1.7 \times 10^{13} \mathrm{~cm}^{-3}$, the spin projection noise of $\mathrm{Cs}$ atom magnetometer is about $1.23 \mathrm{fT} \cdot \mathrm{Hz}^{-1 / 2}$. Therefore, Cs atom magnetometer has the advantages of low noise level, low power consumption, etc. and can be used in many special occasions, thus to have great application prospect. Meanwhile, these researches have provided us with necessary theoretical basis for the experimental study on SERF-Cs atom magnetometer. 


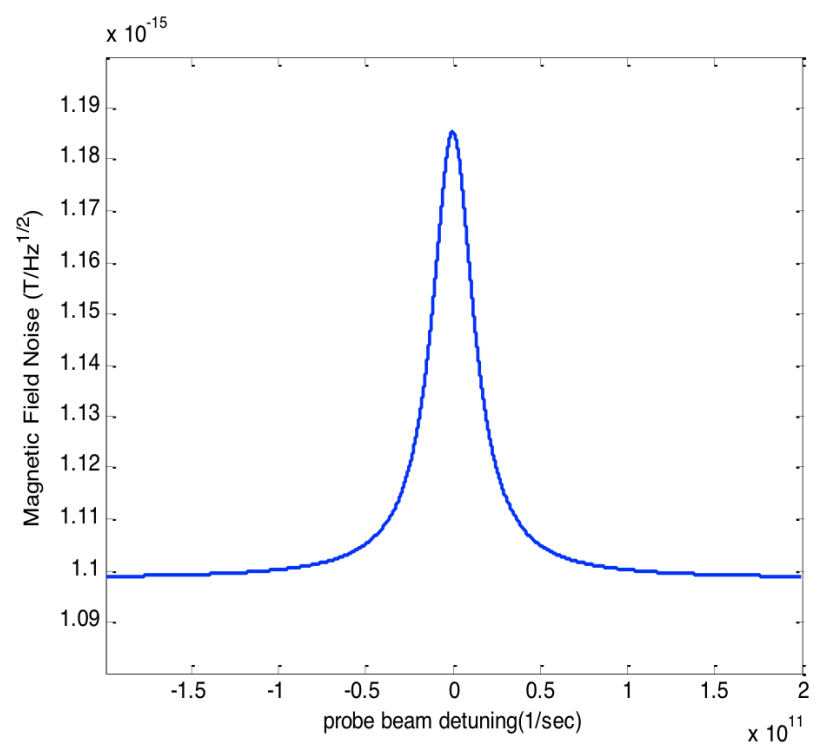

Fig. (3). Relation between Spin Projection Noise of Molecular Density Cs Atom magnetometer and Probe beam detuning.

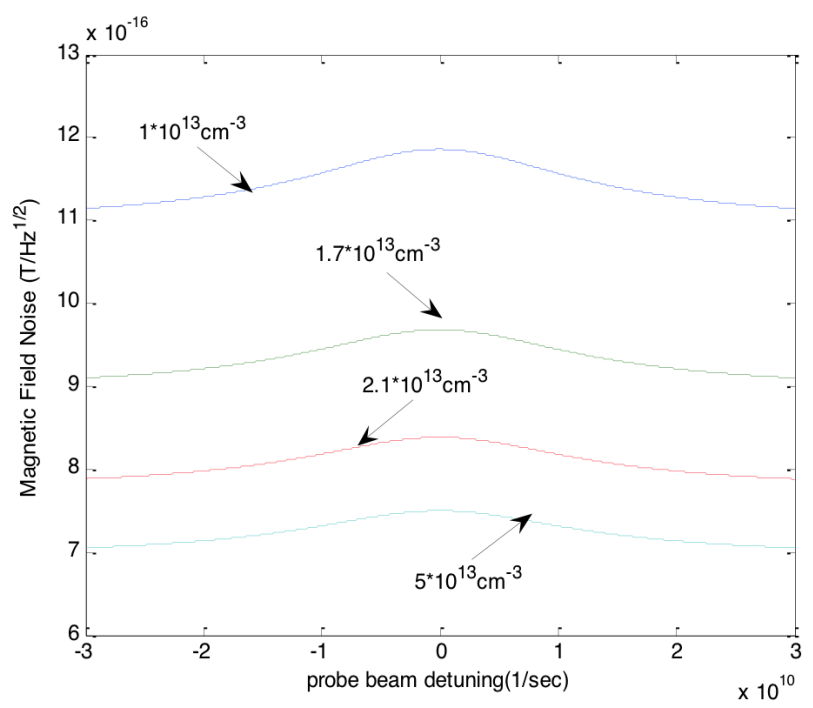

Fig. (4). Relation between Spin Projection Noise of Molecular Density Cs Atom magnetometer and Probe beam detuning.

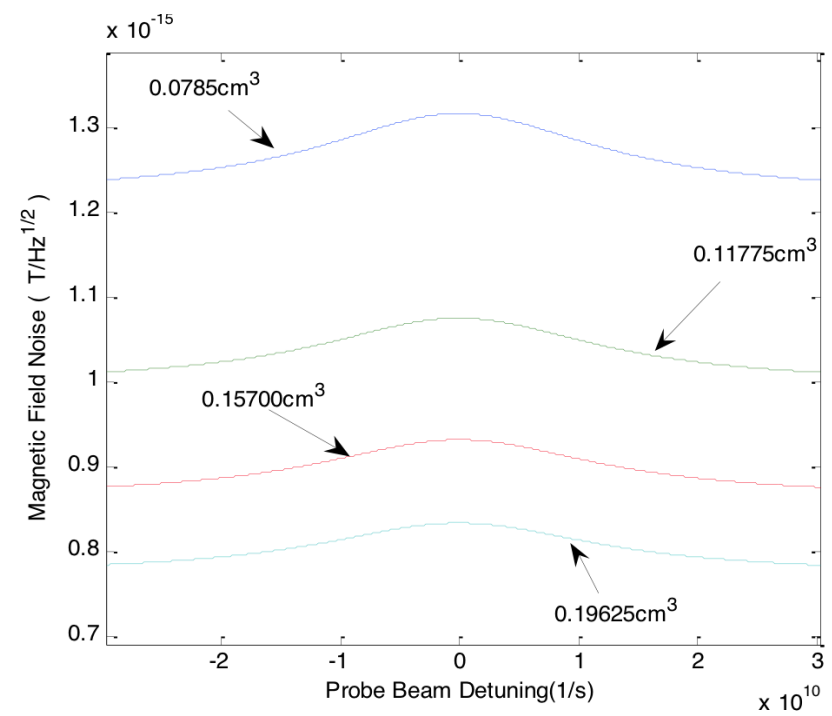

Fig. (5). Relation between Spin Projection Noise of Volume Cs Atom magnetometer and Probe beam detuning. 


\section{CONFLICT OF INTEREST}

The author confirms that this article content has no conflict of interest.

\section{ACKNOWLEDGEMENTS}

This work was part of the Program Supported by Scientific Research Fund of Heilongjiang Provincial Education Department (12533050).

\section{REFERENCES}

[1] I. K. Kominis, "A subfemtotesla multichannel atomic magnetometer", Nature, vol. 422, no. 6932, pp. 596-599, 2003.

[2] F. A. Franz, and C. Volk, "Electronic spin relaxation of the $42 \mathrm{~S}^{1 / 2}$ state of K induced by K-He and K-Ne collisions", Physical Review A, vol. 26, no. 1, pp. 85-92, 1982.

[3] P. G. Killeen, "Exploration trends \& development in 2008", Supplement to the Northern Miner, vol. 95, no. 1, 2009.

[4] J. C. Allred, R. N. Lyman, T. W. Kornack, and M. V. Romalis, "High-sensitivity atomic magnetometer unaffected by spinexchange relaxation ", Physics Review Letter, 23 130801,2002.

[5] I. K. Kominis, T. W. Kornack, and J. C. Allred, "A subfemtotesla multichannel atomic magnetometer", Nature, vol. 422, pp. 596-599, 2003.
[6] D. Budker, "A new spin on magnetometry," Nature, vol. 422, pp. 6932, pp. 574-575, 2003

[7] S. J. Seltzer, and M. V. Romalis, "Unshielded three-axis vector operation of a spin-exchange relaxation-free atomic magnetometer", Applied Physics Letters, vol. 85, no. 20, pp. 4804-4806, 2004.

[8] P. D. D. Schwindt, "Chip-scale atomic magnetometer," Applied Physics Letters, vol. 85, pp. 6409-6411, 2004.

[9] W. C. Griffith, R. Jimenez-Martinez, S. Knappe, J. Kitching, and V. Shah, "Miniature atomic magnetometer integrated with fluxconcentrators," Applied Physics Letters, vol. 94, pp. 023502, 2009.

[10] D. Yu, N. Garcia, and S. Xu, "Toward portable nuclear magnetic resonance devices using atomic magnetometers," Concepts in Magnetic Resonance Part A, vol. 34, no. 2, pp.124-132, 2009.

[11] I. M. Savukov, and M. V. Romalis, "NMR detection with an atomic magnetometer", Physical Review Letters, vol. 94, no. 12, pp. 123001, 2005.

[12] M. P. Ledbetter, I. M. Savukov, and M. V. Acosta, "Spin-Exchange Relaxation Free Magnetrometry with Cs Vapor", Physical Review $A$, vol. 77, pp. 033408, 2008.

[13] D. Budker, and M. Romalis, "Optical magnetometry," Nature Physics, vol. 3, no. 4, pp. 227-234, 2007.

[14] R. Wyllie, "Magnetocardiography with a modular spin-exchange relaxation-free atomic magnetometer array", Physics in Medicine and Biology, vol. 57, no. 9, pp. 2619, 2012.

[15] N. Castagno, "A large sample study of spin relaxation and magnetometric sensitivity of paraffin-coated Cs vapor cells", Applied Physics B, vol. 96, no. 4, pp. 763-772, 2009.

(C) Feng et al.; Licensee Bentham Open.

This is an open access article licensed under the terms of the (https://creativecommons.org/licenses/by/4.0/legalcode), which permits unrestricted, non-commercial use, distribution and reproduction in any medium, provided the work is properly cited. 